\title{
The Role of Fat Emulsion Therapy in a Rodent Model of Propranolol Toxicity: A Preliminary Study
}

\author{
Grant Cave, BHB, MBChBa , Martyn G. Harvey, BHB, MBChB ${ }^{\mathrm{b}}$, Craig D. Castle, MBBS ${ }^{\mathrm{a}}$
}

\author{
a Monash Medical Centre, Melbourne, AU \\ b Waikato Hospital, NZ
}

\begin{abstract}
Introduction: In animal models, lipid emulsion therapy has been shown to ameliorate toxicity from a number of lipid soluble agents. This preliminary study addresses the hypothesis that pretreatment with lipid emulsion protects against propranolol toxicity in rodents.

Methods: Ten spontaneously ventilating Rattus norvegicus rats were pretreated with either lipid emulsion or $0.9 \%$ normal saline before undergoing a constant infusion of propranolol until death. An electrocardiogram (ECG) sampling of heart rate and a QRS duration was performed at two-minute intervals until demise.

Results: There was no significant difference in lethal doses of propranolol between groups. Comparison of percent change in QRS prolongation and heart rate reduction was performed at $60 \%$ of the mean lethal dose in control animals. The percent change in QRS duration was reduced (from -0.9 to $17.3, p=0.016$ ) in the intralipid pretreatment group. Attenuation of propranolol-induced bradycardia observed in the lipid emulsion group approached statistical significance ( $0 \%$ vs. $10.3 \%, p=0.06)$.

Interpretation: The results suggest that lipid emulsion may be effective in ameliorating propranolol toxicity in rats. Previous work gives reason to postulate a pharmacokinetic mechanism for this effect. The results represent encouraging exploratory work, and further work is planned to evaluate the role of lipid emulsion therapy in propranolol toxicity.
\end{abstract}

\section{INTRODUCTION}

Propranolol is a beta blocker commonly involved in poisonings with this class of drug [1]. In addition to its lipophicity, the sodium channel blocking effect of propranolol-when compared to other beta blockers-is thought to contribute to increased mortality [1-3]. The magnitude of sodium channel blockade can be measured experimentally as a blockade of fast inward sodium channels results in a QRS prolongation on the surface ECG (4).

In previous experiments, intravenous lipid emulsions have been shown to ameliorate the toxicity of a number of lipid soluble agents [5-10]. A hypothesized mechanism is the formation of a new intravascular lipid phase that affects drug distribution.

This preliminary study tests the hypothesis that pretreatment with Intralipid ${ }^{\circledR}$ (Pharmacia and Upjohn) 20\%, an isotonic aqueous emulsion of $20 \%$ soybean oil in water, will protect against propranolol toxicity in rats.

\section{Materials and Methods}

Ethical approval was obtained from our local animal ethics committee. 10 female Rattus norvegicus rats (obtained from Ruakura Agresearch, Hamilton, New Zealand) weighing 217-285 grams were studied. The rats were raised in single sex conditions with

Keywords: fat emulsions, intravenous/therapeutic use, beta blockers, adrenergic, propranolol, poisoning Acknowledgements: The authors gratefully acknowledge a research grant from the Monash Medical Centre Emergency Department, and the assistance of Mr. Ric Broadhurst, small animals colony manager, Ruakura Agresearch.

Corresponding author: Dr. Grant Cave, Monash Medical Centre ICU, Clayton Road, Clayton, Melbourne, Australia. Email: grantcave@xtra.co.nz 
TABLE 1. Heart rates prior to and immediately post lipid pretreatment (no propranolol yet infused)

\begin{tabular}{lccc}
\hline & $\begin{array}{c}\text { Baseline heart rate, } \\
\text { beats per minute } \\
\text { (95\%C.I.) }\end{array}$ & $\begin{array}{c}\text { Post lipid pretreatment } \\
\text { heart rate, beats per } \\
\text { minute (95\%C.I.) }\end{array}$ & $\begin{array}{c}\text { p value, intrasubject } \\
\text { differences pre and } \\
\text { post lipid (Wilcoxon } \\
\text { sum of ranks) }\end{array}$ \\
\hline $\begin{array}{l}\text { Intralipid group } \\
\text { Saline group }\end{array}$ & $261(235-286)$ & $240(204-278)$ & 0.13 \\
\hline $\begin{array}{l}\text { p value, intergroup differences } \\
\text { at times indicated } \\
\text { (Mann Whitney) }\end{array}$ & 0.55 & $260(230-288)$ & 0.19 \\
\hline
\end{tabular}

no chance of pregnancy. There was no significant difference in mean weights between the control group (mean $253.4 \mathrm{gm}, 95 \%$ C.I. 222.5 to 284.3 ) and the treatment group (mean $252.4 \mathrm{gm}$, 95\% C.I. 240.3 to 263.6); $(p=0.91)$. Intralipid ${ }^{\circledR}$ was obtained from the Monash Medical Centre pharmacy.

Animals were sedated using our institution's small animals protocol of $50 \mathrm{mg} / \mathrm{kg}$ ketamine and $0.1 \mathrm{mg} / \mathrm{kg}$ xylazine intraperitoneally. Once animals were adequately sedated, tail veins were cannulated with a $24 \mathrm{~g}$ cannula. For the course of the experiment animals were kept on a controlled temperature surface at 37 degrees Celsius.

Ten minutes after the intraperitoneal injection, heart rate sampling was begun using a 3 lead surface ECG at $50 \mathrm{~mm} / \mathrm{sec}$. Heart rates were calculated from a 3 beat interval measured on the rhythm strip to the nearest $0.5 \mathrm{~mm}$.

After the initial ECG samples, animals were pretreated with either $16 \mathrm{ml} / \mathrm{kg} 20 \%$ Intralipid $^{\circledR}$ or $16 \mathrm{ml} / \mathrm{kg} 0.9 \% \mathrm{NaCl}$ infused over 4 minutes. This dose was chosen because it was similar to the dosage used in previous work [7]. Every second experiment was a lipid pretreatment without any blinding of the primary investigator. ECG samplings were repeated following pretreatment. QRS duration was determined by scanning rhythm strips $(50 \mathrm{~mm} / \mathrm{sec})$ at 120 pixels $/ \mathrm{cm}$. These were then magnified until pixelation, each pixel representing $0.00167 \mathrm{sec}$.

Animals were then infused with propranolol at $0.4 \mathrm{mg} / \mathrm{kg} /$ minute. The main outcome variable was the total dose of propranolol to death, defined by the primary investigator as either absence of cardiac electrical activity or cessation of all respiratory effort. Work by Toet et al $[11,12]$ established that the mode of death in spontaneously ventilating rats infused with propranolol is respiratory depression.

Further analysis of data from the work of Toet et al $[11,12]$ showed that there were no significant differences in heart rate and blood pressure between ventilated and nonventilated rodents when $60 \%$ of mean lethal dose was used. Therefore, we chose as secondary outcome variables the heart rate and QRS duration when $60 \%$ of the mean lethal dose for control animals had been infused.

Data were analyzed using Graphpad Prism $4^{\circledR}$ (GraphPad Software, San Diego, CA). Statistical significance was set at a 2-tailed $\mathrm{p}$ value $<0.05$. The Mann-Whitney test was used for intergroup comparisons. Wilcoxon sum of ranks was used to compare intrasubject differences.

\section{RESULTS}

There were 5 rats in each of the Intralipid ${ }^{\circledR}$ and saline pretreated groups. No significant difference was observed in heart rate or QRS duration following pretreatment [Table 1].

The mean survival time during propranolol infusion in the Intralipid ${ }^{\circledR}$ pretreated group was 47 minutes: $(18.8 \mathrm{mg} / \mathrm{kg}$ total infused); (95\% C.I. 4.5 to $89.75 \mathrm{~min})$. The mean survival time in the saline pretreated group was 18.75 minutes: $(7.5 \mathrm{mg} / \mathrm{kg}$ total infused); (95\% C.I. 14.25 to $23 \mathrm{~min})$. The difference was not significant $(\mathrm{p}=0.15)$. Respiratory arrest was the mode of death in all subjects.

Compared to values at commencement of propranolol infusion, an analysis of percent change in QRS duration and heart rate at $4.8 \mathrm{mg} / \mathrm{kg}$ infused (12 minutes) suggested benefit to intralipid pretreatment on heart rate reduction, and a significant lessening of QRS duration prolongation [Table 2]. This point was chosen as the sampling time most representative of $60 \%$ of the mean lethal dose of propranolol in controls, and all study animals survived to this point.

\section{Discussion}

These results suggest that lipid emulsion may be effective in ameliorating propranolol toxicity in rats. An increase in a lethal dose of propranolol in the lipid-pretreated animals was found, but the observed difference did not reach statistical significance.

TABLE 2. Percent changes in heart rate and QRS duration from commencement of propranolol infusion to $4.8 \mathrm{mg} / \mathrm{kg}$ infused.

\begin{tabular}{lcc}
\hline & $\begin{array}{c}\text { Percent reduction } \\
\text { heart rate }(\mathbf{9 5 \%} \text { C.I.) }\end{array}$ & $\begin{array}{c}\text { Percent change } \\
\text { QRS duration } \\
\text { (95\% C.I.) }\end{array}$ \\
\hline Intralipid group & $0(-9.3$ to 9.3$)$ & $-0.9(-4.7$ to 2.9$)$ \\
\hline Saline group & $10.3(2.0$ to 18.5$)$ & $17.3(7.2$ to 23.2$)$ \\
\hline P value (Mann Whitney) & 0.06 & 0.016 \\
\hline
\end{tabular}


The best use for this data will be in powering future studies. Assuming an effect as large as those demonstrated in this study, the same distribution parameters, and normality for survival time distributions, 27 animals would be needed in each arm to provide $80 \%$ power of demonstrating an effect on survival times at the 0.05 significance level.

There is a wide distribution of survival time in lipidpretreated animals, as evidenced by the 95\% C.I. that was notably wider than controls. One hypothetical explanation is that Intralipid $^{\circledR}$ may have both protective effects on toxicity of propranolol, and it may have some independent toxic effects. Further studies may use a lipid only arm (no propranolol post pretreatment) to further evaluate this issue. Of note, a similar wide variability in effect of lipid pretreatment on bupivacaine toxicity in rats was noted [7]. A strong effect for lipid emulsion in treating bupivacaine toxicity was later shown in dogs [6].

The effects of lipid emulsion were favorable on cardiac parameters measured at $60 \%$ of the mean lethal dose. We used our control data and analysis of previous work $[11,12]$ to establish $4.8 \mathrm{mg} / \mathrm{kg}$ as the most appropriate point for analysis of heart rate and QRS duration in our protocol. Because these endpoints were established retrospectively, they must be considered secondary outcomes.

Attenuation of propranolol-induced bradycardia in the fat emulsion group was observed at $4.8 \mathrm{mg} / \mathrm{kg}$, which approached statistical significance. In previous animal studies, Intralipid ${ }^{\circledR}$ administration has been associated with metabolic effects resulting in tachycardia $[14,15]$. The non-significant reduction in heart rate post Intralipid ${ }^{\circledR}$ pretreatment suggests these metabolic effects were not the cause for the protective effect of lipid. Further studies (using a lipid only arm) would be needed to properly evaluate the cause for the protective effect of lipid.

A significant effect preventing QRS duration prolongation suggests intralipid pretreatment protected against the local anaesthetic effects of propranolol. Although other mechanisms for QRS prolongation from propranolol-such as beta blockade or a central depressant effect [16]—could be forwarded, they would not alter the interpretation of the data showing beneficial effect for Intralipid ${ }^{\circledR}$ on this parameter.

Previous work has demonstrated a beneficial role for fat emulsion therapy in the setting of lipid soluble drug toxicity. A pharmacokinetic mechanism has been hypothesized to explain these effects [5-10]. The most impressive report to date involves a canine model of bupivicaine-induced cardiac arrest. In this study, all six dogs treated with lipid emulsion after 10 minutes of cardiac arrest were successfully resuscitated. In the control group, no dogs were successfully resuscitated [6]. Postulated mechanisms for observed effects of lipid emulsions on lipid soluble drug toxicity include creation of an intravascular lipid phase acting either as a drug depot or a conduit to metabolism or redistribution [5-10].

This study has a number of limitations: principally small numbers and an effect on total lethal dose that is not statistically significant. The principal investigator was not blinded to pretreatment. However, endpoints were objective, decreasing the potential for bias. While there is some evidence that ventilating rats would not have altered cardiac parameters at the point they were analysed $[11,12]$, control of respiratory status with ventilation would have been preferable.

\section{Interpretation}

These results suggest lipid emulsion may be effective in ameliorating toxicity of propranolol in rats. Previous work provides a plausible pharmacokinetic mechanism to explain the beneficial effect.

The results represent encouraging exploratory work, and further work is planned to elucidate the role of lipid emulsion in treating propranolol toxicity.

\section{REFERENCES}

1. Roth B. Toxicity, beta blocker. Emedicine [Updated 2005 Apr 15]. Available from: http://www.emedicine.com/emerg/ topic59.htm

2. Love JN, Howell JM, Litovitz TL, Klein-Schwartz W. Acute beta blocker overdose: factors associated with the development of cardiovascular morbidity. J Toxicol Clin Toxicol 2000; 38: 275-81.

3. Reith DM, Dawson AH, Epid D, et al. Relative toxicity of beta blockers in overdose. J Toxicol Clin Toxicol 1996; 34: 273-8.

4. Kolecki P, Curry S. Poisoning by sodium channel blocking agents. Critical Care Clinics 1997; 13: 829-848.

5. Krieglstein J, Meffert A, Niemeyer D. Influence of emulsified fat on chlorpromazine availability in rat blood. Experientia 1974; 30: 924-926.

6. Weinberg G, Pipper B, Feinstein d, Hoffman W. Lipid emulsion infusion rescues dogs from bupivacaine-induced cardiac toxicity. Reg Anesth Pain Med. 2003; 28:198-202.

7. Weinberg G, VadeBoncouer T, Ramaraju G, Garcia-Amaro M, Cwik M. Pretreatment or resuscitation with a lipid infusion shifts the dose response to bupivacaine induced asystole in rats. Anesthesiology 1998; 88: 1071-1075.

8. Goor Y, Goor O, Cabili S. A lipid emulsion reduces mortality from clomipramine overdose in rats. Vet Hum Toxicol 2002; 44: 30.

9. Tiphine M, Letsher-Bru V, Herbrecht R. Amphotericin B and its new formulations: pharmacologic characteristics, clinical efficacy and tolerability. Transpl Infect Dis 1999; 1: 273-278.

10. Straathof D, Driessen O, Meijer W, Van Rees H, Vermeu P, Vermeu T. Influence of intralipid infusion on elimination of phenytoin. Arch Int Pharmacodyn 1984; 267: 180-186.

11. Toet A, Biestook JD, Vleeming W, Wemer W, Meulenbelt J, de Wilt DJ. Reduced survival after isoprenaline/dopamine in d,1 propranolol intoxicated rats. Human and Experimental Toxicology 1996; 15: 120-128.

12. Toet A, Wemer J, Vleeming W, Biesebeek JD, Meulenbelt J, de Wilt DJ. Experimental study of the detrimental effect of 
dopamine/glucagon combination in d,l propranolol intoxication. Human and Experimental Toxicology 1996; 15: 411-21.

13. Masuda Y, Matsuoka H. Chronotropic response to cardiac sympathetic nerve stimulation in spontaneously hypertensive rats. Can J Physiol Pharmacol 1997; 75: 97-103.

14. Van de Melde M, Wouters P, Rolf N, Van Aken H, Flameng $\mathrm{W}$, Vandermeersch E. Long chain triglycerides improve recovery from myocardial stunning in conscious dogs. Cardiovascular Research 1996; 32: 1008-1015.
15. Chelly J, Oguchi T, Liang C, Hartley C, Doursout M. Role of the intralipid solvent in propofol induced nitric oxide stimulation. Anesthesiology 1996; 85: A555.

16. Thomas R, Behbehani M, Coyle D, Densen D. Cardiovascular toxicity of local anesthetics: an alternative hypothesis. Anesth Analg 1986; 65: 444-450.

17. Minton N, Goode A, Henry J. The effect of a lipid suspension on amitriptyline disposition. Arch Toxicol 1987; 60: 46769. 